\title{
Partial frequency band gap in one-dimensional magnonic crystals
}

\author{
M. Kostylev ${ }^{1, a)}$ P. Schrader, ${ }^{1}$ R. L. Stamps, ${ }^{1}$ G. Gubbiotti, ${ }^{2}$ G. Carlotti, ${ }^{2}$ A. O. Adeyeye, ${ }^{3}$ \\ S. Goolaup, ${ }^{3}$ and N. Singh ${ }^{3}$ \\ ${ }^{1}$ School of Physics, University of Western Australia, Crawley, Western Australia 6009, Australia \\ ${ }^{2}$ CNISM, Dipartimento di Fisica, Università di Perugia, Perugia 1-06123, Italy \\ ${ }^{3}$ Information Storage Materials Laboratory Department of Electrical and Computer Engineering, \\ National University of Singapore 117576, Singapore
}

(Received 15 January 2008; accepted 11 March 2008; published online 1 April 2008)

\begin{abstract}
Collective spin wave modes propagating in an array of magnetic stripes coupled by dynamic dipole interaction are investigated by Brillouin light scattering. It is demonstrated that this structure supports propagation of discrete spin waves at any angle with respect to the stripes length. The data are interpreted using a theoretical model based on the Bloch wave approach. It is shown that, due to the one-dimensional artificial periodicity of the medium, the gaps in the spin wave spectrum are partial: the frequency passbands for propagation along the direction of periodicity overlap with the stop bands for propagation along the stripes. (C) 2008 American Institute of Physics.
\end{abstract}

[DOI: $10.1063 / 1.2904697$ ]

The development of magnetic recording technologies based on patterned media requires a deep understanding of the magnetic dynamics of laterally confined structures on a submicron length scale. When the desired time response is in the subnanosecond regime, ${ }^{1,2}$ the switching behavior of the magnetic elements is determined by the frequency and damping time of spin wave excitations.

Standing-spin-wave resonances in patterned arrays of magnetic wires and dots were extensively studied in the last years (Refs. 3 and 4 and references therein.) Discrete resonance frequencies ("dispersionless modes"), which are formed due to spin wave quantization in the direction of lateral confinement, have been observed in several experiments. ${ }^{5}$ Very recently, we performed Brillouin light scattering (BLS) measurements of spin waves in longitudinally magnetized array of magnetic stripes of rectangular cross section. ${ }^{6}$ The distance between the adjacent stripes was chosen to be large enough to exclude dynamic dipole coupling between the nearest neighbors. When the transferred wavevector was along the stripes long axis ( $y$ direction), several propagating spin wave modes were detected, characterized by a continuous wavevector component along the stripe (determined by the wavevector conservation law in the magnon-photon interaction) and discrete values of the component along the second in-plane direction, i.e., along the stripes short axis ( $x$ direction). On the other hand, we also carried out a preliminary study of spin waves in a dense array of longitudinally magnetized wires, coupled by dipolar interaction. These spin waves, whose wavevector was perpendicular to the stripes, have been described as collective excitations of a one-dimensional (1D) magnonic crystal, ${ }^{7,8}$ with a periodic dispersion encompassing several Brillouin zones, associated with the artificial spatial periodicity of the structure.

In this work, by measuring the frequencies of collective spin wave modes for propagation at different in-plane angles with the $y$ axis and by a theoretical modeling, we aim to investigate the band structure of $1 \mathrm{D}$ magnonic crystals as a a) Author to whom correspondence should be addressed. Electronic mail:
kostylev@cyllene.uwa.edu.au. function of the in-plane wavevector components, with special attention paid to the existence of partial bandgap.

The sample under investigation is a 1D periodic array of interacting Permalloy $\left(\mathrm{Ni}_{80} \mathrm{Fe}_{20}\right)$ stripes (width: $w=350 \mathrm{~nm}$, thickness: $L=30 \mathrm{~nm}$; edge-to-edge spacing: $\Delta=55 \mathrm{~nm}$, and structure period $T=w+\Delta=405 \mathrm{~nm}$ ). The sample was fabricated on a silicon substrate using deep ultraviolet lithography with $248 \mathrm{~nm}$ exposure wavelength followed by a lift-off technique. ${ }^{9}$

BLS measurements were carried out at the GHOST laboratory (University of Perugia). ${ }^{10}$ Monochromatic $p$-polarized light from a solid state laser $(\lambda=532 \mathrm{~nm})$ with a power of about $200 \mathrm{~mW}$, was focused on the patterned area using a camera objective of $f$-number 2 and focal length of $50 \mathrm{~mm}$. Cross-polarized, backscattered light was analyzed using a Sandercock-type (3+3)-pass tandem Fabry-Pérot interferometer. ${ }^{11}$ In our measurement, the incidence angle of light (against the sample normal) was kept constant and equal to $\theta=30^{\circ}$ which corresponds to a value of transferred wavevector $k=(4 \pi / \lambda) \sin (\theta)=1.81 \times 10^{5} \mathrm{rad} / \mathrm{cm}^{-1}$. A fixed

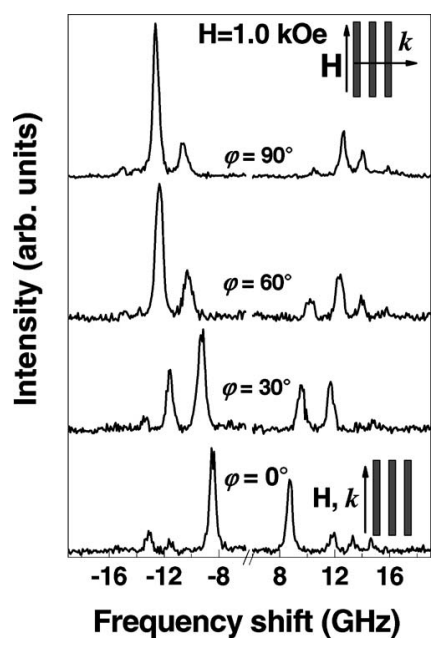

FIG. 1. Sequence of Brillouin light scattering spectra measured for $H=1.0 \mathrm{kOe}$ at different in-plane angles $(\varphi)$ between the stripes length ( $y$ axis) along which $H$ is applied and the scattering plane. Insets show the scattering geometries for $\varphi=0^{\circ}$ and $\varphi=90^{\circ}$. 


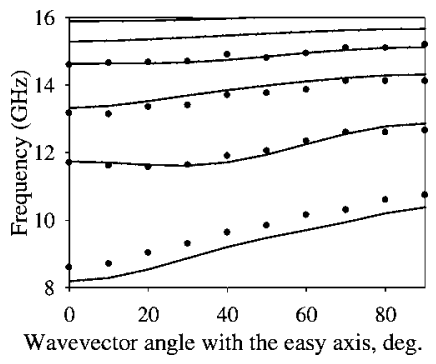

FIG. 2. Dependence of collective mode frequency on the wave propagation angle $\varphi$ with the major array $y$ axis. Dots: experiment, solid lines: numerical calculation.

external magnetic field $H=1.0 \mathrm{kOe}$ was applied parallel to the major axis of the stripes ( $y$ axis) and BLS measurements were done changing the "in-plane angle" $\varphi$ between the scattering plane and the $y$ axis. A sequence of measured BLS spectra measured in such conditions is shown in Fig. 1 and the spin wave dispersion $\omega(\varphi)$ derived from the spectra is shown in Fig. 2. One sees a well-resolved set of modes whose frequency increases with the angle $\varphi$.

In addition, BLS spectra were also measured by changing the incidence angle $\theta$ when both $H$ and $k$ are parallel to the stripes length $(\varphi=0)$. For these latter measurements, the applied field was $H=0.5 \mathrm{kOe}$ and the corresponding frequencies are shown in Fig. 3 together with the experimental data, relative to the case $\varphi=90^{\circ}$, taken from Ref. 8 .

To explain dependence of the spin wave eigenfrequencies on the wave number we constructed a theory of collective spin wave modes propagating at an arbitrary angle $\varphi$ with the array major stripe $y$ axis. The collective spin wave modes are formed due to magnetostatic interaction which couples the dynamical magnetization of adjacent stripes resonating into individual stationary states. Our theory is based on the Bloch wave approach for collective modes developed in Refs. 12 and 13 and Green's function of dipole field of guided spin waves with continuous wavevectors along the $y$ axis. ${ }^{6}$ The $1 \mathrm{D}$ calculation of Ref. 6 was overcome here by the use of a two-dimensional Green's function which allows one to rigorously account for eventual nonuniformity of the out-of-plane $(z)$ profile of the dynamic magnetization. Including the dependence of the Green's function on the $z$ coordinate is important since the aspect ratio $p=L / w$ for the stripes used in our experiment is about 0.1 . Therefore, effective dipole pinning of magnetization ${ }^{14}$ at the upper and lower surfaces of the stripes may be important resulting in a nonnegligible inhomogeneity of the $z$ profile of the dynamic

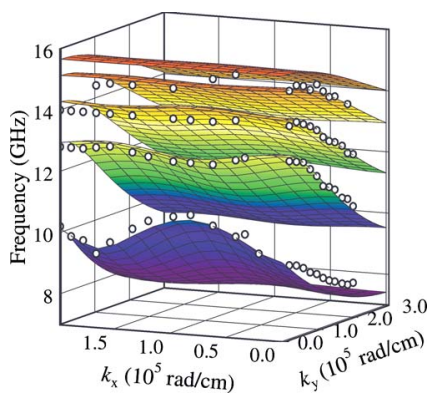

FIG. 3. (Color online) Spectrum of collective modes in the two-dimensional space $k_{x}, k_{y}$. Surfaces are calculations and dots are experimental points measured as a function of $\theta$ for $\varphi=0^{\circ}$ and $\varphi=90^{\circ}$. These latter data were taken from Ref. 8. magnetization. Indeed, the z-resolved calculations below give more reasonable fitting values for the saturation magnetization and the gyromagnetic ratio for Permalloy than obtained in the 1D calculation of Ref. 6.

The stripe dynamic magnetization $\mathbf{m}$ and the collective dynamic dipole field inside the stripes $\mathbf{h}$ is cast in the form

$$
\begin{aligned}
& \mathbf{m}(y, x, z, t)=\mathbf{m}_{k_{x} k_{y}}(x, z) \exp \left(i k_{y} y+i k_{x} x-i \omega_{k_{x} k_{y}} t\right), \\
& \mathbf{h}(y, x, z, t)=\mathbf{h}_{k_{x} k_{y}}(x, z) \exp \left(i k_{y} y+i k_{x} x-i \omega_{k_{x} k_{y}} t\right),
\end{aligned}
$$

where $\mathbf{m}_{k_{x} k_{y}}(x, z)$ and $\mathbf{h}_{k_{x} k_{y}}(x, z)$ are periodical functions: $\mathbf{m}_{k_{x} k_{y}}(x, z)=\mathbf{m}_{k_{x} k_{y}}(x+T, z), \mathbf{h}_{k_{x} k_{y}}(x, z)=\mathbf{h}_{k_{x} k_{y}}(x+T, z)$, and $T$ is the lattice period. The transverse wave number $k_{x}=k \sin (\varphi)$ and the longitudinal wave number $k_{y}=k \cos (\varphi)$ can take continuous values.

Based on Green's function formalism (see, e.g., Ref. 14), in a continuous-medium approximation, one obtains an expression for the dipole field of a macrospin chain with arbitrary variation of magnetization along the $y$ axis. The chain is assumed to be of infinite length in the direction $y$ and of infinitely small width in the directions $x$ and $z$. From this expression, one easily finds components of Green's function

$$
G_{k_{y} \alpha \alpha^{\prime}}(x, z)=\frac{\partial}{\partial \alpha} \frac{\partial}{\partial \alpha^{\prime}} K_{0}\left[k_{y} \sqrt{\left(x-x^{\prime}\right)^{2}+\left(z-z^{\prime}\right)^{2}}\right],
$$

where $x^{\prime}$ and $z^{\prime}$ are the coordinates of the chain, $x$ and $z$ are the coordinates where the magnetic field of the chain is observed, $K_{0}$ is the zeroth order modified Bessel function of the second kind, and $\alpha$ and $\alpha^{\prime}$ are $x$ or $z$ and $x^{\prime}$ or $y^{\prime}$, respectively. Green's function $\hat{G}_{k_{y}}$ is then generalized to include space periodicity along the $x$ direction. ${ }^{12}$ For our array of stripes, the tensorial Green's function $\hat{G}_{k_{x} k_{y}}\left(x, x^{\prime}, z, z^{\prime}\right)$ in the reduced scheme takes the form

$$
\begin{aligned}
\hat{G}_{k_{x} k_{y}}\left(x, x^{\prime}, z, z^{\prime}\right)= & \sum_{n=-\infty}^{\infty} \hat{G}_{k_{y}}\left(x, x^{\prime}-n T, z, z^{\prime}\right) \\
& \times \exp \left[i k_{x}\left(x^{\prime}-n T-x\right)\right] \exp \left(-|n| T / l_{c}\right) .
\end{aligned}
$$

The coordinate $x$ and $x^{\prime}$ takes the values inside one stripe, i.e., from $x, x^{\prime}=0$ to $x, x^{\prime}=w$. Using Eq. (1), the relation between $\mathbf{m}_{k_{x} k_{y}}(x, z)$ and $\mathbf{h}_{k_{x} k_{y}}(x, z)$ takes the form

$$
\mathbf{h}_{k_{x} k_{y}}(x, z)=4 \pi \int_{0}^{w} \int_{0}^{L} \hat{G}_{k_{x} k_{y}}\left(x, x^{\prime}, z, z^{\prime}\right) \mathbf{m}_{k_{x} k_{y}}\left(x^{\prime}, z^{\prime}\right) d z^{\prime} d x^{\prime} .
$$

The term $\exp \left(-|n| T / l_{c}\right)$ has been phenomenologically introduced to Eq. (3). It accounts for a possibly finite coherence length $l_{c}$ of the collective modes in the $x$ direction.

In this work, we numerically solve Eq. (4) together with the linearized Landau-Lifshitz magnetic torque equation

$$
\begin{aligned}
-i \omega_{k_{x} k_{y}} \mathbf{m}_{k_{x} k_{y}}(x, z)= & -|\gamma|\left\{\mathbf{M}_{0} \times\left[\mathbf{h}_{k_{x} k_{y}}(x, z)+\mathbf{h}_{k_{x} k_{y}}^{\mathrm{ex}}(x, z)\right]\right. \\
& \left.-\mathbf{H} \times \mathbf{m}_{k_{x} k_{y}}(x, z)\right\},
\end{aligned}
$$

where $\mathbf{h}_{k_{x} k_{y}}^{\mathrm{ex}}$ is the effective dynamic exchange field. The solution results in dependence of the collective mode eigenfrequency $\omega_{\mathbf{k}}$ on the modulus $k$ and the direction $\varphi$ of the total in-plane wavevector $k: k=\sqrt{k_{x}^{2}+k_{y}^{2}}, \varphi=a \tan \left(k_{x} / k_{y}\right)$. Results 
of the numerical calculation for $k=1.81 \times 10^{5} \mathrm{rad} / \mathrm{cm}$ are shown in Fig. 2 by the solid lines, showing a good agreement with the experimental data. To produce these results we used the following magnetic parameters of Permalloy: saturation magnetization $4 \pi M_{0}=10100 \mathrm{G}$, gyromagnetic ratio $|\gamma| / 2 \pi=2.82 \times 10^{6} \mathrm{~Hz} / \mathrm{Oe}$ and exchange constant $A=9$ $\times 10^{-7} \mathrm{erg} / \mathrm{cm}$. To obtain this good fit, we had to assume that the coherence length of the Bloch waves is finite and equal to two structure periods: $l_{c}=2 T$.

To achieve a better understanding of the band structure of the 1D magnonic crystal under investigation, we performed a calculation of the full two-dimensional eigenspectrum of collective modes $\omega_{\mathbf{k}}\left(k_{x}, k_{y}\right)$, shown in Fig. 3. This calculation was done for a value of the applied field $H=0.5 \mathrm{kOe}$ in order to compare the results of calculation with both the present experiment performed as a function of $\theta$ for $\varphi=0^{\circ}$, and our previous experimental results, for $\varphi=90^{\circ}$, of Ref. 8 (shown by dots). One sees a set of surfaces which does not cross each other. It is noticeable, however, that the spectrum does not show full stop bands: the stop bands existing for a fixed value of either $k_{y}$ or $k_{x}$ overlap with the permitted frequencies for other values of the two parameters, thus, one concludes that the frequency gaps in this structure are partial.

Let us now discuss in more details the main characteristics of the dynamical excitations.

(a) For $k_{x}=0 \quad(\varphi=0)$, the collective modes represent a modification of the lowest-order backward volume magnetostatic mode (BVMSW) of a continuous film since the field is collinear with the wavevector. The slope of the frequency sheets is in fact negative, as expected for BVMSW. Due to the effective dipole pinning of magnetization at the stripe boundaries, ${ }^{14}$ the distribution of the dynamical magnetization across the stripe width $w$ is inhomogeneous (see Ref. 6). This causes an increase of the slopes of the dispersion curves for all the collective modes with respect to that of the lowest-order BVMSW in a continuous film. Our calculations show that the maximum effective pinning and, consequently, the maximum slope is obtained for totally uncoupled stripes $(\Delta \rightarrow \infty)$. Dipole coupling of adjacent stripes reduces the effective pinning of magnetization at the stripe edges making the $x$ profile of dynamic magnetization more homogeneous and reducing the slope of the dispersion curves. The difference in slope with the continuous film decreases with decrease in $\Delta$ and for $\Delta=0$, one eventually retrieves the dispersion for a continuous film of the same thickness $L$.

(b) For $k_{y}=0 \quad\left(\varphi=90^{\circ}\right)$, the collective modes represent coupled resonances of Damon-Eshbach magnetostatic surface wave (MSSW). In the case of dipole coupling of such resonances a Bloch wave is formed, characterized by Brillouin zones induced by the artificial periodicity. The lowest resonance has a quasihomogeneous distribution of the dynamic magnetization across the stripe width, i.e., very similar to MSSW in a continuous film. Therefore, the initial slope of the lowest mode is positive and takes the maximum value. The higherorder collective modes have either a positive or a negative slope depending on the specific parity of the dy- namic magnetization profile across the stripe widths. Similar to the lowest mode, those modes with an even number of nodes in the profile possess a net dynamic magnetic moment $\overline{\mathbf{m}}=\int_{0}^{w} \int_{0}^{L} \mathbf{m}_{k_{x} k_{y}}(x, z) d z d x$ and have a positive slope. The modes with an odd number of nodes have a vanishing $\overline{\mathbf{m}}$ and a negative slope.

Rotation of the vector $k$, with fixed modulus, from $\varphi=0$ to $\varphi=90^{\circ}$, as we did in the experiment (see Figs. 1 and 2), corresponds to transition from a modified BVMSW geometry to a modified MSSW geometry. This results in a moderate increase in frequency of all the collective modes, as seen in Fig. 2, as well as to a change of the dispersion slope of the even modes (possessing a nonvanishing $\overline{\mathbf{m}}$ ) from negative to positive.

Finally, we note that the experimental values of wave numbers in Fig. 3 are spatial frequencies of Fourier spectrum of the $x$ profile of the dynamic magnetization ${ }^{5}$ and the theoretical ones are Bloch wavevectors. However, this is the general property of Bloch wave dispersion that in the extended scheme, the latter is equal to the former.

In conclusion, we showed that plane periodical structures of parallel dipole-coupled magnetic stripes support propagation of Bloch waves at any angles with the major structure axis. Due to lateral confinement within the stripe width, the eigenwaves spectrum has a large number of modes, while the artificial periodicity induces the presence of Brillouin zones and consequent periodicity in $k$ space. Noticeably, the frequency gaps in the spectrum are partial, i.e., the stop bands for propagation along the major stripe axis and the frequency passbands for propagation perpendicular to it overlap. This is connected to the fact that the artificial magnonic crystal studied here is $1 \mathrm{D}$ while the occurrence of a full band gap would require at least two-dimensional periodicity.

Support by Australian Research Council and MIUR (Italy) is gratefully acknowledged.

${ }^{1}$ R. L. Stamps and B. Hillebrands, Appl. Phys. Lett. 75, 1143 (1999).

${ }^{2}$ Th. Gerrits, H. A. M. van den Berg, J. Hohlfeld, L. Bär, and Th. Rasing, Nature (London) 418, 509 (2002).

${ }^{3}$ C. Bayer, J. Jorzick, B. Hillebrands, S. O. Demokritov, R. Kouba, R. Bozinoski, A. N. Slavin, K. Guslienko, D. Berkov, N. Gorn, and M. P. Kostylev, Phys. Rev. B 72, 064427 (2005).

${ }^{4}$ Magnetic Properties of Laterally Confined Nanometric Structures, edited by G. Gubbiotti (Transworld Research Network, Kerala, India, 2006).

${ }^{5}$ C. Mathieu, J. Jorzick, A. Frank, S. O. Demokritov, A. N. Slavin, B. Hillebrands, B. Bartenlian, C. Chappert, D. Decanini, F. Rousseaux, and E. Cambril, Phys. Rev. Lett. 81, 3968 (1998).

${ }^{6}$ M. P. Kostylev, G. Gubbiotti, J.-G. Hu, G. Carlotti, T. Ono, and R. L. Stamps, Phys. Rev. B 76, 054422 (2007).

${ }^{7}$ G. Gubbiotti, S. Tacchi, G. Carlotti, P. Vavassori, N. Singh, S. Goolaup, A. O. Adeyeye, A. Stashkevich, and M. Kostylev, Phys. Rev. B 72, 224413 (2005).

${ }^{8}$ G. Gubbiotti, S. Tacchi, G. Carlotti, N. Singh, S. Goolaup, A. O. Adeyeye, and M. Kostylev, Appl. Phys. Lett. 90, 092503 (2007).

${ }^{9}$ C. C. Wang, A. O. Adeyeye, N. Singh, Nanotechnology 17, 1629 (2006).

${ }^{10}$ See (http://ghost.fisica.unipg.it).

${ }^{11}$ J. R. Sandercock, in Light Scattering in Solids III, edited by M. Cardona and G. Güntherodt (Springer, Berlin, 1982), p. 173.

${ }^{12}$ M. P. Kostylev, A. A. Stashkevich, and N. A. Sergeeva, Phys. Rev. B 69, 064408 (2004).

${ }^{13}$ C. Bayer, M. P. Kostylev, and B. Hillebrands, Appl. Phys. Lett. 88, 112504 (2006)

${ }^{14}$ K. Yu. Guslienko, S. O. Demokritov, B. Hillebrands, and A. N. Slavin, Phys. Rev. B 66, 132402 (2002). 
Applied Physics Letters is copyrighted by the American Institute of Physics (AIP). Redistribution of journal material is subject to the AIP online journal license and/or AIP copyright. For more information, see http://ojps.aip.org/aplo/aplcr.jsp 